\title{
Tunable Semiconducting Polymer Nanoparticles with INDT-Based Conjugated Polymers for Photoacoustic Molecular Imaging
}

Thomas Stahl, ${ }^{\dagger}$ Robin Bofinger, ${ }^{\ddagger}$ Ivan Lam, ${ }^{\ddagger}$ Kealan J. Fallon, ${ }^{\ddagger}$ Peter Johnson, ${ }^{\S}$ Olumide Ogunlade, ${ }^{\dagger}$ Vessela Vassileva, ${ }^{\S, \mathbb{I}}$ R. Barbara Pedley, ${ }^{\S}$ Paul C. Beard, ${ }^{\dagger}$ Helen C. Hailes, ${ }^{\ddagger}$ Hugo Bronstein, ${ }^{*}, \ddagger$ and Alethea B. Tabor*,+(0)

${ }^{\dagger}$ Department of Medical Physics and Biomedical Engineering, University College London, Gower Street, London WC1E 6BT, United Kingdom

${ }^{\ddagger}$ Department of Chemistry, University College London, 20, Gordon Street, London WC1H 0AJ, United Kingdom

${ }^{\S}$ UCL Cancer Institute, Paul O’Gorman Building, 72 Huntley Street, London WC1E 6DD, United Kingdom

Supporting Information

\begin{abstract}
Photoacoustic imaging combines both excellent spatial resolution with high contrast and specificity, without the need for patients to be exposed to ionizing radiation. This makes it ideal for the study of physiological changes occurring during tumorigenesis and cardiovascular disease. In order to fully exploit the potential of this technique, new exogenous contrast agents with strong absorbance in the near-infrared range, good stability and biocompatibility, are required. In this paper, we report the formulation and characterization of a novel series of endogenous contrast agents for photoacoustic imaging in vivo. These contrast agents are based on a recently reported series of indigoid $\pi$-conjugated organic semiconductors, coformulated with 1,2-dipalmitoyl-sn-glycero-3-phosphocholine, to give semiconducting polymer nanoparticles of about $150 \mathrm{~nm}$ diameter. These

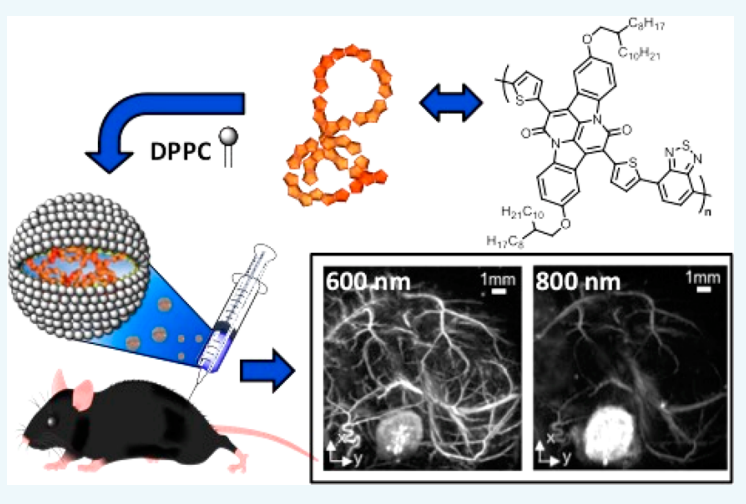
nanoparticles exhibited excellent absorption in the near-infrared region, with good photoacoustic signal generation efficiencies, high photostability, and extinction coefficients of up to three times higher than those previously reported. The absorption maximum is conveniently located in the spectral region of low absorption of chromophores within human tissue. Using the most promising semiconducting polymer nanoparticle, we have demonstrated wavelength-dependent differential contrast between vasculature and the nanoparticles, which can be used to unambiguously discriminate the presence of the contrast agent in vivo.
\end{abstract}

\section{INTRODUCTION}

Photoacoustic (PA) imaging is an emerging technique based on the use of laser generated ultrasound, which holds great promise for visualizing anatomical structures and physiological changes in vivo. It combines the advantages of ultrasound imaging (submillimeter spatial resolution with deep tissue imaging penetration) with the high contrast and specificity of optical imaging. ${ }^{1}$ It is noninvasive and does not require the use of ionizing radiation, and has significant potential for the clinical and preclinical study of conditions such as breast, head and neck, melanoma, colorectal, prostate, and ovarian cancers, and cardiovascular disease. ${ }^{2}$ Endogenous PA image contrast is based on optical absorption provided by naturally occurring chromophores, such as lipids or hemoglobin, the latter enabling exquisite images of the vasculature to be acquired. ${ }^{3}$ However, many cells and tissues are weakly absorbing at visible and nearinfrared wavelengths and thus require labeling with exogenous contrast agents to provide PA image contrast. Such exogenous contrast agents would ideally have a strong extinction coefficient, good photostability, high thermodynamic efficiency, narrow absorption spectrum, and low toxicity, and be selectively retained at the target while being rapidly cleared from the rest of the body. Most importantly, the ideal exogenous contrast agent would absorb in the near-infrared range (NIR), i.e., $620-920 \mathrm{~nm}$. This range is known as the optical window of tissue, due to the low absorption of water and hemoglobin in this region.

Various types of contrast agents may be used and a range of small molecule NIR dyes is already available; however these have several disadvantages. These include modest molar extinction coefficients, photoinstability after prolonged irradiation, and a tendency to aggregate. Moreover, their small size contributes to a rapid systemic clearance, reducing the likelihood of target delivery and retention. ${ }^{2}$ Nanoparticle contrast agents, which include gold nanorods (GNR) and single walled carbon nanotubes (SWCNT), have several

Received: April 4, 2017

Revised: $\quad$ May 13, 2017

Published: May 31, 2017 
advantages: they exhibit very high extinction coefficients, the absorption wavelength may be tuned, they can carry additional cargoes such as therapeutic drugs, and they accumulate in targets such as tumors via the EPR effect. ${ }^{4}$ However, concerns about high cost, poor biodegradability, and potential toxicity of gold- and carbon-based nanoparticles have recently prompted research into other types of nanoparticle contrast agents. Semiconducting polymer nanoparticles (SPNs) have recently been developed; these are formulated from $\pi$-conjugated organic semiconductors along with amphiphilic polymers or surfactants to produce nanoparticles that are stable in aqueous solutions. The enhanced photostability, high quantum yield, and biocompatibility ${ }^{5}$ of the resulting SPNs has already led to their use in various biological imaging and biosensing applications. ${ }^{6,7}$ It has recently been demonstrated that SPNs, formulated using $\pi$-conjugated organic semiconductors with high NIR absorption, can be used as PA contrast agents. ${ }^{8-11}$ These SPNs have been reported to have significantly stronger signal per mass, and better photostability, than GNR or SWNT; preliminary studies have shown these to be effective for in vivo imaging of reactive oxygen species $^{8}$ and brain vascular imaging.

Clearly a major advantage of using SPNs as PA contrast agents would be the potential to tune the PA properties of these nanoparticles by using NIR $\pi$-conjugated organic semiconductors with different structural and spectral properties. However, to date only a limited selection of suitable polymers has been investigated, and as a result, the previously reported SPNs have UV absorption peaks at a correspondingly limited set of single wavelengths. Of particular interest is the synthesis of high extinction coefficient, narrow band gap conjugated polymers allowing for efficient generation of a PA signal in the near-infrared region of the electromagnetic spectrum.

In this paper, we report the formulation and full characterization of a novel series of $\pi$-conjugated organic semiconductor nanoparticles with outstanding properties for the use as contrast agents for in vivo PA imaging. These nanoparticles exhibit strong absorption in the NIR, with good PA signal generation efficiencies and photostability. Their extinction coefficient is up to three times higher compared to similar particles previously reported. ${ }^{8-11}$ In addition, the absorption maximum of the novel nanoparticles is conveniently located in the spectral region of low absorption of chromophores within human tissue, making the particles well suited as contrast agents for PA imaging. Furthermore, the novel family of $\pi$ conjugated organic semiconductors that have been used to formulate the SPNs can be readily tuned to a variety of NIR wavelengths by small variations in the electron richness or deficiency of the component monomers. This has allowed us, in this work, to produce a family of SPN PA contrast agents that can be tuned to different wavelengths in the biologically relevant NIR window.

\section{RESULTS AND DISCUSSION}

Preparation and Characterization of NIR $\pi$-Conjugated Organic Semiconductors. We previously reported the synthesis of a conjugated polymer ${ }^{12}$ with an extremely narrow band gap, the origin of which was the exceptional electron accepting properties of the little used 2,9-dihydroxy7,14-di(thiophen-2-yl)-diindolo[3,2,1-de: $\left.3^{\prime}, 2^{\prime}, 1^{\prime}-i j\right][1,5]$ naphthyridine-6,13-dione (INDT) core. Using this novel chromophore a family of indigoid ultranarrow band gap materials with excellent solution processability were synthesized by copolymerization with monomers of varying electron richness or deficiency. ${ }^{13}$ The four polymers which were chosen for this study were the previously reported PCPDTBT $\mathbf{1}^{6-12}$ and the indigoid $\pi$-conjugated organic semiconductors INDT-T 2, INDT-S 3, and INDT-BT 4 (Figure 1).

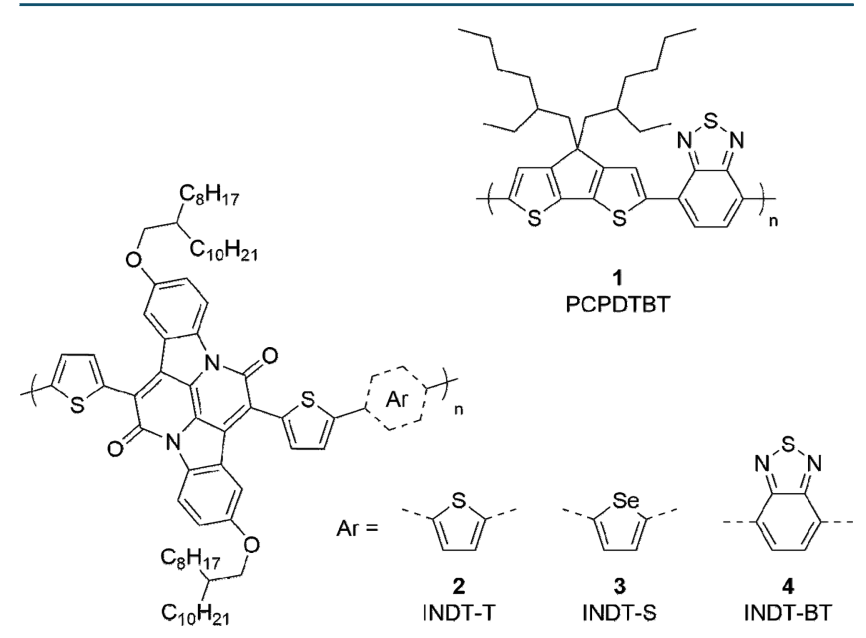

Figure 1. Structures of the $\pi$-conjugated organic semiconductors PCPDTBT 1, INDT-T 2, INDT-S 3, and INDT-BT 4.

All of these display band-gaps in the region of $\sim 1.2 \mathrm{eV}$, making them ideal candidates for PA imaging in comparison to other wider band gap materials such as PCPDTBT 1. In addition, the absorption maxima of INDT-T 2, INDT-S 3, and INDT-BT 4 are significantly red-shifted in comparison to PCPDTBT 1 (Figure 2).

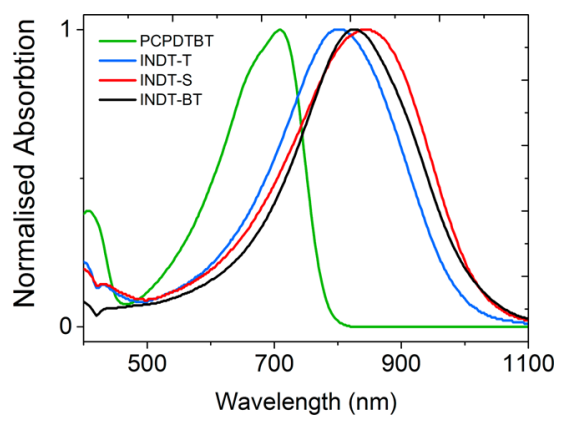

Figure 2. UV-visible spectra of the $\pi$-conjugated organic semiconductors PCPDTBT 1, INDT-T 2, INDT-S 3, and INDT-BT 4 in chlorobenzene.

Formulation and Characterization of SPNs. Two approaches are currently used to formulate SPNs, nanoprecipitation, and mini-emulsion. ${ }^{6,7}$ In the nanoprecipitation approach, the $\pi$-conjugated organic semiconductor is dissolved in a "good" solvent and then added to an excess of a "poor" solvent under ultrasonic dispersion; the change in solvent polarity results in the aggregation of the polymers. The two solvents must be miscible with each other, such as acetone/ water, THF/water, or ethanol/water. This is then followed by evaporation of the organic solvent in an inert atmosphere to give the SPNs in an aqueous solution. However, for organic semiconductors that are not soluble in organic solvents that are miscible with water, the mini-emulsion approach is employed. In this method, the organic semiconductor is dissolved in a solvent such as chloroform, which is immiscible with water, and 
added to a dispersion of surfactant in water under ultrasonic dispersion. In this work we aimed to coencapsulate the $\pi$ conjugated organic semiconductors with a saturated lipid, 1,2dipalmitoyl-sn-glycero-3-phosphocholine (DPPC) (Figure 3), as it has been previously shown that varying the particle surface by using different lipids has little effect on the PA properties of the resulting SPNs.

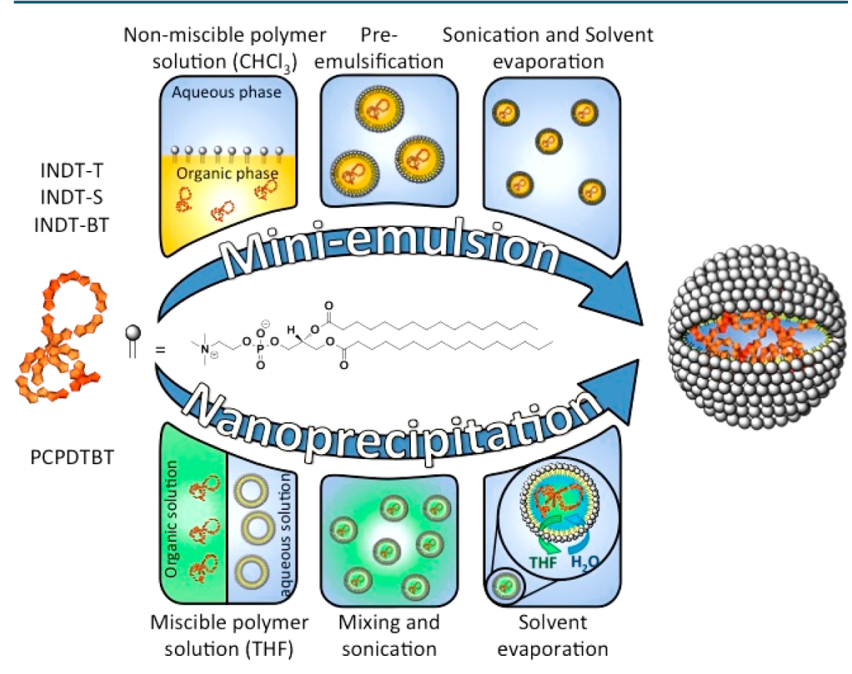

Figure 3. Schematic showing the preparation of the SPNs by nanoprecipitation or mini-emulsion approaches.

Using the nanoprecipitation method, as previously reported, ${ }^{8}$ an aqueous solution of SPN1 was successfully prepared from PCPDTBT and DPPC. However, polymers INDT-T, INDT-S, and INDT-BT are not fully soluble in THF, and for these polymers the mini-emulsion approach was used to give aqueous solutions of SPN2, SPN3, and SPN4 (Table 1). The nanoparticles prepared were between 120 and $160 \mathrm{~nm}$ in diameter. This makes them ideally sized for cancer imaging, as nanoparticles between 100 and $200 \mathrm{~nm}$ in size accumulate in tumors $^{14}$ through a combination of leaky tumor endothelium and ineffective lymphatic drainage, a phenomenon known as the enhanced permeability and retention effect (EPR). The measured $\zeta$-potentials varied between $-25.6 \mathrm{mV}$ and -37.8 $\mathrm{mV}$.

The PA properties of the aqueous solutions of the nanoparticles SPN1-4 were determined using a custom designed PA spectroscope. ${ }^{15}$ PA amplitude and PA derived extinction spectra were generated over the 450-950 nm spectral range and the thermalization efficiency (the efficiency for the conversation from absorbed light to heat) was calculated as described previously. ${ }^{15}$ A UV/vis/NIR spectrophotometer (PerkinElmer 750s) was used to obtain the absorbance spectrum of the nanoparticles for comparison with the PA spectra. The resulting spectra with the measured extinction coefficient are shown in Figure $4 \mathrm{a}$ and values of $\lambda_{\max }$ and $\varepsilon$ summarized in Table 1 . The nanoparticles did not display any detectable fluorescence as would be expected from their extremely narrow band gap (due to the energy gap law) indicating that radiative decay is not a loss mechanism in these materials.

In order to investigate the photostability of the nanoparticles freshly prepared samples of SPN1-4 were irradiated with $18 \times$ $10^{3}$ laser pulses, while the PA amplitude was recorded every minute, averaging over 100 signals. For these experiments the laser output was tuned to the wavelength of peak absorption of the individual samples. In the case of SPN1 the peak absorption wavelength cannot be generated by the laser used and therefore the laser was tuned to $750 \mathrm{~nm}$ for the bleaching experiment. For comparison two common organic dyes (cresyl violet and IR 820) were analyzed for their photostability under the same conditions. The results of the photobleaching experiments are shown in Figure $4 b$, showing high photostability of all of the nanoparticles. Irrespective of the formulation method, nanoparticles of consistent and similar size and zeta potential were formed, with strong absorption in the NIR, high photostability, and thermalization efficiencies of $100 \%$. The latter indicates that the nanoparticles exhibit negligible radiative relaxation with a quantum yield close to zero. Due to the superior extinction coefficient $\left(253 \mathrm{~cm}^{-1} \mathrm{~L} \mathrm{~g}^{-1}\right)$ and the spectral position of the wavelength of peak absorption at $800 \mathrm{~nm}$, SPN4 was chosen for further in vivo experiments.

In Vitro Cytotoxicity Assessment. In vitro cytotoxicity of SPN1, SPN2, SPN3, and SPN4 was evaluated in the 293T human embryonic kidney cell line. Cells were exposed to a range of concentrations $(0-25 \mu \mathrm{g} / \mathrm{mL})$ of the nanoparticles for $24 \mathrm{~h}$, and cell viability was determined by the MTT assay. There was no significant effect on cell viability with any of the nanoparticles at the investigated range of concentrations (Figure 5), indicating that the nanoparticles are biocompatible in vitro.

In Vivo PA Imaging. In vivo PA images were acquired of a bolus of SPN4, injected subcutaneously into the flank of a mouse, as described in the Experimental Procedures section. Figure 6 shows a selection of PA images at 5 wavelengths out of 10 different wavelengths acquired, of the same field of view (14 $\mathrm{mm} \times 14 \mathrm{~mm} \times 6 \mathrm{~mm}$ ), before (Figure 6a) and after injection of SPN4 (Figure 6b), revealing the presence of the nanoparticles after injection. The images in Figure $6 \mathrm{~b}$ were acquired sequentially, within $1.5 \mathrm{~h}$ after injection. At $600 \mathrm{~nm}$, blood provides stronger optical absorption than SPN4, resulting in relatively low image contrast of the nanoparticles, whereas at $800 \mathrm{~nm}$ SPN4 has a stronger optical absorption compared to the absorption of blood. The contrast from SPN4 was still visible at long wavelengths $(1000 \mathrm{~nm})$, against the background of blood and increasing absorption water. The visible areas of higher contrast within the injected bolus of Figure $6 \mathrm{~b}$ are due to

Table 1. Physical and Spectroscopic Properties of the Four $\pi$-Conjugated Organic Semiconductors and the Semiconducting Polymer Nanoparticles SPN1-4 Formulated from Them

\begin{tabular}{|c|c|c|c|c|c|c|c|c|c|}
\hline polymer & $\lambda_{\max }{ }^{a}[\mathrm{~nm}]$ & $M_{\mathrm{n}}[\mathrm{kDa}]$ & $M_{\mathrm{w}} / M_{\mathrm{n}}$ & SPN & diameter $[\mathrm{nm}]$ & PDI & $\zeta$ potential $[\mathrm{mV}]$ & $\lambda_{\max }[\mathrm{nm}]$ & $\varepsilon\left[\mathrm{cm}^{-1} \mathrm{~g} \mathrm{~L}^{-1}\right]$ \\
\hline РСРDТВТ 1 & 709 & 6 & 1.3 & SPN1 & 145 & 0.2 & -37.8 & 700 & 83 \\
\hline INDT-T 2 & 802 & 20 & 4.0 & SPN2 & 159 & 0.25 & -25.6 & 780 & 79 \\
\hline INDT-S 3 & 844 & 25 & 3.3 & SPN3 & 120 & 0.23 & -29.8 & 790 & 139 \\
\hline INDT-BT 4 & 826 & 40 & 3.0 & SPN4 & 160 & 0.25 & -31.0 & 800 & 253 \\
\hline
\end{tabular}

${ }^{a} \lambda_{\max }$ measured in chlorobenzene solution. 
(a)
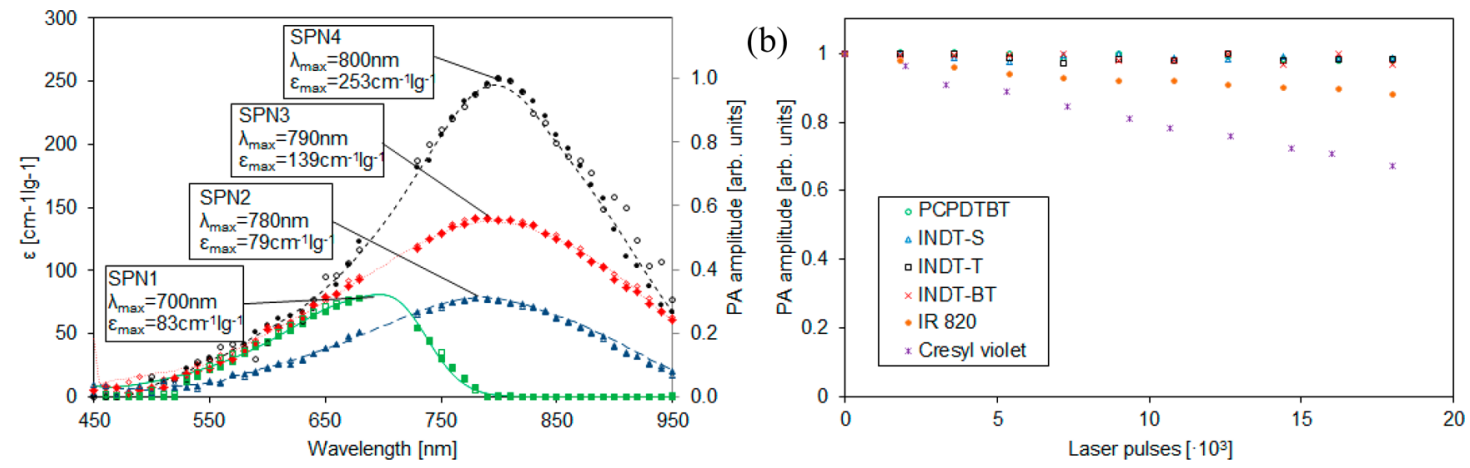

Figure 4. (a) Extinction spectra of SPN1-4, measured using the PA spectroscope (normalized PA amplitude spectra: hollow markers; PA derived extinction spectra: solid markers) and spectra obtained using a spectrophotometer (lines). (b) Photostability of SPN1-4 at concentrations of $\sim 40$ $\mathrm{mg} \mathrm{L}^{-1}$, cresyl violet $(700 \mu \mathrm{M})$, and IR $820(230 \mu \mathrm{M})$ under continuous irradiation with pulsed laser light with a fluence of $\sim 2 \mathrm{~mJ}^{-2}$. Wavelengths were chosen corresponding to the wavelength of peak absorption of the individual nanoparticles, except for SPN1 where $750 \mathrm{~nm}$ was chosen as the excitation wavelength.

SPN1

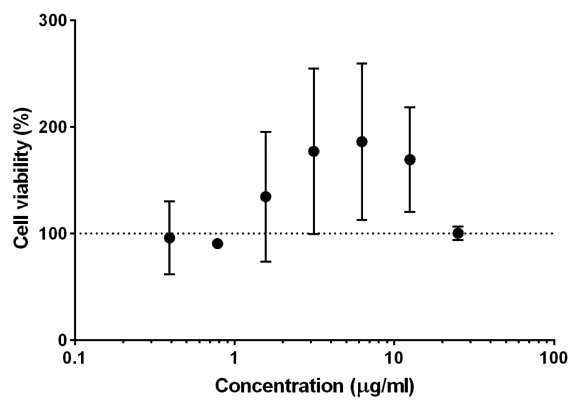

SPN3

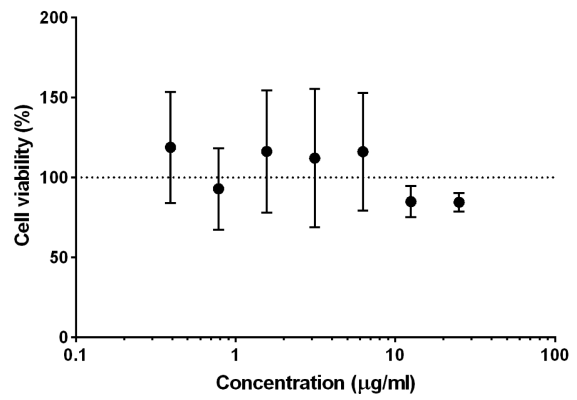

SPN2

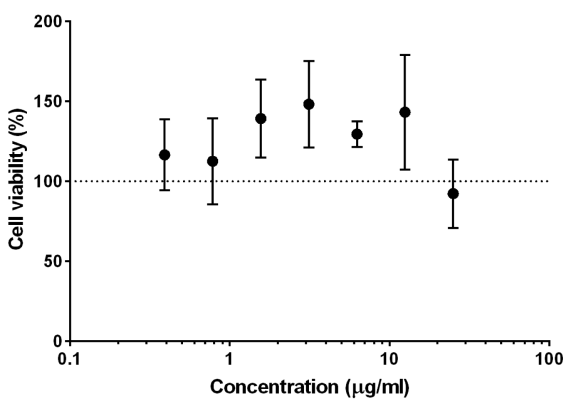

SPN4

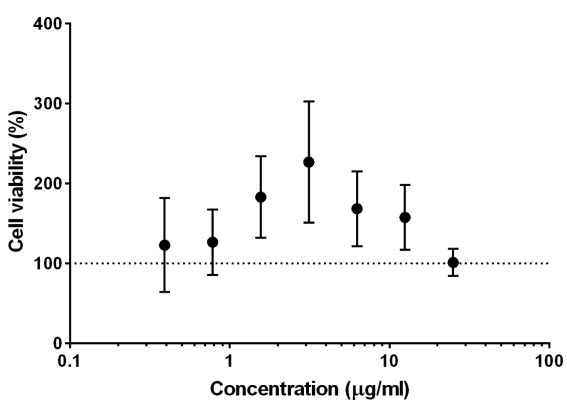

Figure 5. Evaluation of the cytotoxicity in 293T human embryonic kidney cells of SPN1, SPN2, SPN3, and SPN4 as determined using the MTT assay following a $24 \mathrm{~h}$ incubation period (data presented as mean $\pm \mathrm{SD}$ ).

aggregation of SPN4 during subcutaneous injection. Figure 6c shows the in vivo wavelength dependence of SPN4, derived from the in vivo images, some of which are shown in Figure $6 \mathrm{~b}$.

These data points were obtained by integrating the image intensity in $3 \mathrm{D}$ over the regions corresponding to the nanoparticles normalized by the integrated image intensity of the first slice of the $3 \mathrm{D}$ image where the particles are not present. This normalization step corrects for the difference in pulse energy output of the laser at the different wavelengths although it does not correct for spectral variations in the subsurface fluence at the nanoparticle bolus. As evidenced by the close agreement of the in vivo and in vitro PA spectra in Figure $6 c$, these variations are small due to the superficial location of the bolus. Also shown in Figure $6 \mathrm{c}$ are the normalized specific absorption coefficient spectra of oxy- hemoglobin $(\mathrm{HbO} 2)$ and deoxyhemoglobin ( $\mathrm{HHb})$. These results illustrate the wavelength-dependent differential contrast between the vasculature and SPN4, which can be used to unambiguously discriminate the presence of SPN4 in vivo.

\section{CONCLUSIONS}

In this paper we describe the chemistry, formulation, and PA characteristics of a novel series of semiconducting polymer nanoparticles which we have developed as PA contrast agents. These are based on novel indigoid $\pi$-conjugated organic semiconductors with a high extinction coefficient, narrow band gap, and with absorption maxima located in the near-infrared in the "optical window" of low absorption of endogenous chromophores within human tissue. These polymers were coformulated with DPPC to give stable nanoparticles of 

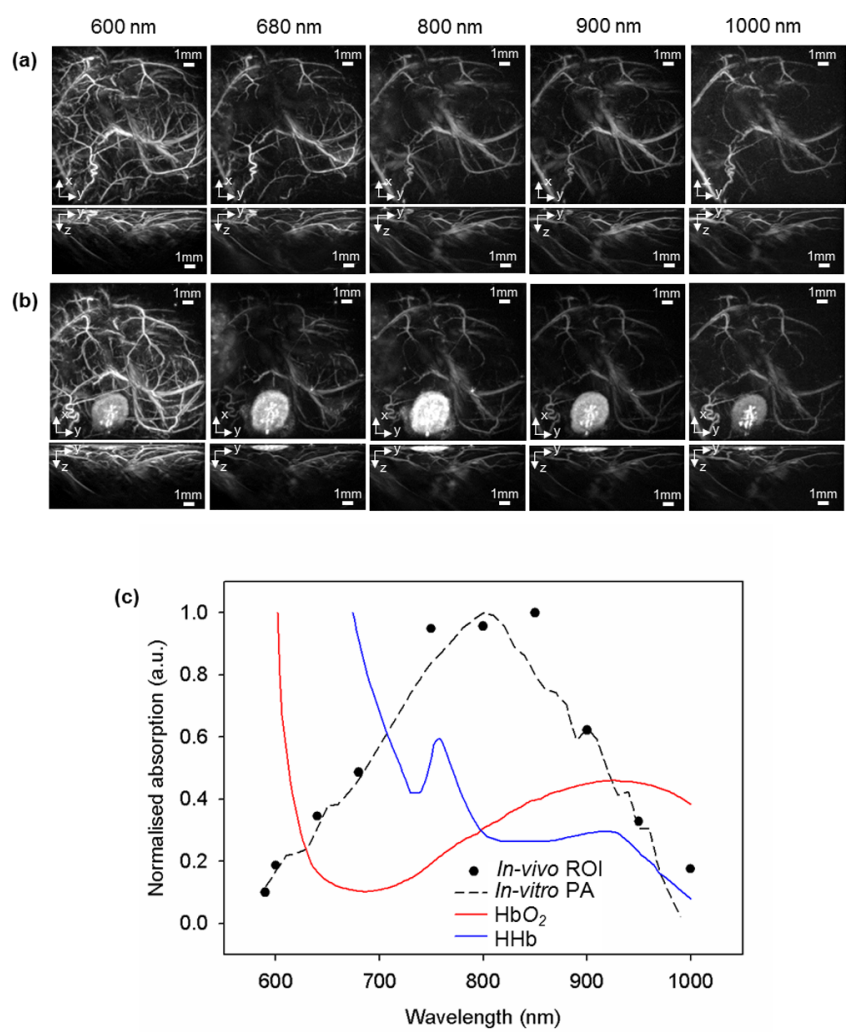

Figure 6. Multiwavelength in vivo photoacoustic showing spectral dependence of INDT-BT nanoparticles (SPN4) and vasculature: Subsample of photoacoustic images (a) before and (b) after subcutaneous injection of a $10 \mu \mathrm{L}$ solution, containing $1.6 \mu \mathrm{g}$ of SPN4, into the flank of a SCID mouse. $x$ - $y$ MIPs (top rows, area $14 \times$ $14 \mathrm{~mm}^{2}$ ) and $y-z$ MIPs (bottom rows, area $14 \times 6 \mathrm{~mm}^{2}$ ) acquired at different excitation wavelengths are shown. (c) Normalized wavelength dependence of SPN4 absorption from the in vivo photoacoustic images shown in (b), normalized in vitro PA spectra of SPN4 and the normalized specific absorption of oxyhemoglobin and deoxyhemoglobin.

consistent size and zeta potential, good photoacoustic signal generation efficiencies, high photostability, and extinction coefficients up to three times higher than previously reported SPN. Moreover, our studies demonstrated no significant in vitro cytotoxicity in human embryonic kidney cells. This is in line with previous reports indicating that similar semiconducting polymer nanoparticles have low toxicity, ${ }^{5,9,10}$ making these tunable nanoparticles excellent candidates for in vivo imaging, and suggesting that other organic semiconductors should also be investigated for such applications.

In preliminary in vivo experiments we have demonstrated that at $800 \mathrm{~nm}$ the best of the nanoparticles, SPN4, provides a significantly stronger signal than adjacent blood vessels, allowing us to unambiguously image the presence of SPN4 relative to the vasculature. We have also shown that small modifications to this series of $\pi$-conjugated organic semiconductors allows us to tune the absorption maxima of the various SPN to different wavelengths. This will in turn allow the SPN to be tuned to wavelengths where relatively inexpensive lasers are available. It will also make it possible to select SPNs which are optimized for greatest tissue penetration, which might be different for different tissue, organs, and pathologies. Finally, the tuneability of these SPN will eventually enable multiplexing, with two or more SPN, each with a different absorption spectrum and targeted to different receptors being used to visualize multiple targets simultaneously. While the spectral characteristics of the reported SPN will be sufficient for most applications, given the broad spectral features of endogenous chromophores, for applications requiring multiple contrast agents with different spectra it will be highly desirable to reduce the breadth of the spectrum and make the onset of the absorption steeper. These are current issues in the general field of organic electronics, and this suggests an important avenue for future research in the area of semiconducting polymer nanoparticles. Ultimately, for these SPN to be effective as contrast agents for preclinical and clinical use, issues of biocompatibility, stability, and validation of targeting must also be studied, ${ }^{16}$ and in future work we will address these issues.

\section{EXPERIMENTAL PROCEDURES}

Chemicals. 1,2-Dipalmitoyl-sn-glycero-3-phosphocholine (DPPC) was purchased from Avanti Lipids, USA. Poly[cyclopentadithiophene-alt-benzothiadiazole] (PCPDTBT 1) was purchased from Sigma-Aldrich. All other chemicals were obtained from Sigma-Aldrich unless otherwise stated. The synthesis and characterization of INDT-T 2, INDT-S 3, and INDT-BT 4 have been reported elsewhere, ${ }^{13}$ and are included in the Supporting Information.

Preparation of SPNs. SPN1 was prepared via nanoprecipitation using PCPDTBT $\left(M_{\mathrm{w}} 20872 \mathrm{~g} \mathrm{~mol}^{-1}\right)$ which was dissolved in THF at a concentration of $0.25 \mathrm{~g} \mathrm{~L}^{-1}$. The formation of nanoparticles using the nanoprecipitation method was initiated via the rapid injection, with a syringe, of $1 \mathrm{~mL}$ of the polymer solution into $9 \mathrm{~mL}$ of deionized water under continuous sonication using a probe sonicator (Q125; $3 \mathrm{~mm}$ tip diameter; QSonica, US) set to 6W RMS for $30 \mathrm{~s}$. Subsequently, $1 \mathrm{~mL}$ of a solution of 1,2-dipalmitoyl-sn-glycero-3-phosphocholine (DPPC) in THF:water (2:3) was injected to the sonicated solution in order to stabilize the nanoparticles. The mixture was kept under sonication for an additional $1 \mathrm{~min}$ at $6 \mathrm{~W}$ RMS before the sonicator-tip was removed from the mixture. For the final step the organic solvent was evaporated via heating the resulting solution in a water-bath to $45{ }^{\circ} \mathrm{C}$ and bubbling nitrogen through it. After about $2 \mathrm{~h}$ the resulting suspension of SPN1 was then filtered through a $0.22 \mu \mathrm{m}$ poly(ether sulfone) syringe driven filter (Merck Millipore, US) and washed three times with deionized water using centrifugal filters with an MWCO of 30k Da (Merck Millipore, US) under centrifugation at $4000 \mathrm{rpm}$ for $3 \mathrm{~min}$ at $4{ }^{\circ} \mathrm{C}$. After the washing step the nanoparticles were resuspended in deionized water using an ultrasonication bath. The resulting nanoparticles appear blue when in suspension and were analyzed using DLS, TEM, spectrophotometer, and PA spectroscopy.

Polymers based on INDT-x have little or low solubility in any other organic solvent than dichloromethane and chloroform. Consequently, the mini-emulsion approach was applied for the preparation of SPN2-4. Therefore, $2.5 \mathrm{mg}$ of DPPC were dispersed in $2.5 \mathrm{~mL}$ of deionized water and vortexed for $1 \mathrm{~min}$ before the mixture was sonicated for about $3 \mathrm{~min}$ in a sonicator bath. The polymers (INDT-T 2, INDT-S 3, and INDT-T 4) were dissolved in $\mathrm{CHCl}_{3}$ to obtain a final concentration of $0.6 \mathrm{~g}$ $\mathrm{L}^{-1}$ of each of the polymers in the organic solvent. To ensure the full solvation of the polymers, each of the polymer mixtures was heated to reflux to obtain dark blue solutions. Subsequently, $160 \mu \mathrm{L}$ of one of the dissolved polymers was pre-emulsified in the aqueous surfactant mixture by stirring 
with a magnetic stirrer for $5 \mathrm{~min}$ at $1000 \mathrm{rpm}$. Next, the emulsion was subjected to high power sonication (10W RMS) for $30 \mathrm{~s}$ using a probe sonicator. After formation of the miniemulsion, the power output of the probe sonicator was reduced to $5 \mathrm{~W}$ RMS and the mixture subjected to cycles of $30 \mathrm{~s}$ sonication and $10 \mathrm{~s}$ of resting with the probe sonicator turned off. After about $5 \mathrm{~min}$, the opaque solution began to clear, resulting in a clear colored solution. Then, the solution was placed in a water-bath of $60{ }^{\circ} \mathrm{C}$ for about $2 \mathrm{~h}$ in order to evaporate the remaining organic solvent. Lastly, the aqueous suspension of polymeric nanoparticles was filtered through a $0.22 \mu \mathrm{m}$ poly(ether sulfone) syringe driven filter and washed three times with deionized water using centrifugal filters with an MWCO of $30 \mathrm{kDa}$ under centrifugation at $4000 \mathrm{rpm}$ for $3 \mathrm{~min}$ at $4{ }^{\circ} \mathrm{C}$. After the washing step the nanoparticles were resuspended in deionized water using an ultrasonication bath.

Polymer and Nanoparticle Characterization. UV-vis spectra were recorded on a PerkinElmer Lambda 950 spectrophotometer between 400 and 1100 at $2 \mathrm{~nm}$ steps. Solution spectra were recorded in chlorobenzene and thin-films were spin-coated at $10000 \mathrm{rpm}$ for $30 \mathrm{~s}$ onto glass substrates using polymer solutions of $5 \mathrm{mg} / \mathrm{mL}$ in chlorobenzene. Number-average $\left(M_{\mathrm{n}}\right)$ and weight-average $\left(M_{\mathrm{w}}\right)$ molecular weights of all four polymers were determined using gel permeation chromatography in chlorobenzene at $80{ }^{\circ} \mathrm{C}$ against a polystyrene standard using an Agilent Technologies 1200 series spectrometer. The size and zeta potential of nanoparticles SPN1-4 were measured using a Malvern Zetasizer Nano (ZSZEN3600, Malvern Instruments Ltd., UK), using a $\mathrm{He}-\mathrm{Ne}$ laser $633 \mathrm{~nm}$, Max $4 \mathrm{~mW}$ for excitation and a detection angle of $173^{\circ}$.

PA Spectroscopy. The PA spectroscope ${ }^{15}$ used for the characterization of the nanoparticles consists of a $30 \mathrm{~Hz}$ tunable fiber-coupled Nd:YAG pumped OPO laser (Spitlight 600, InnoLas Laser $\mathrm{GmbH}$, Krailling, Germany) with a pulse-topulse tuning capability as the excitation source and a PVDF transducer with a $-3 \mathrm{~dB}$ bandwidth of $<20 \mathrm{MHz}$. Irradiating a sample contained in a Perspex sample cuvette generates acoustic signals which are detected by the PVDF transducer, amplified and finally digitized using a data-acquisition card. As described previously, ${ }^{15}$ PA spectra were generated by scanning the excitation laser in the spectral region between 450 and 950 $\mathrm{nm}$ in $5 \mathrm{~nm}$ step increments and acquiring the PA signals at each wavelength step. The PA amplitude spectrum was obtained from this data by plotting the normalized peak positive amplitudes of the detected PA signals as a function of wavelength to provide a relative spectrum. The extinction coefficient spectra were obtained from the same PA data set by fitting an exponential to the initial compressive part of the detected PA signals to recover the absorption coefficient $\mu_{\mathrm{a}}$. The extinction coefficient was then obtained by dividing $\mu_{\mathrm{a}}$ by the concentration. For an estimate of the concentration of the nanoparticles prepared, the ratio of the weight of the polymers injected to the aqueous phase during the procedure and the final volume of the nanoparticle solution was used.

In Vitro Cytotoxicity of Nanoparticles. The human embryonic kidney cell line, 293T, was cultured in Dulbecco's Modified Eagle Medium (DMEM) containing 10\% fetal bovine serum at $370 \mathrm{C}$ in a humidified environment, containing $5 \%$ $\mathrm{CO}_{2}$. In vitro cytotoxicity of the nanoparticles was evaluated by the MTT [3-(4,5-dimethylthiazol-2-yl)-2,5-diphenyltetrazolium bromide] cell viability assay.
Briefly, $5 \times 10^{4}$ cells were seeded in 96-well plates and incubated overnight. Cells were then exposed to a range of concentrations $(0-25 \mu \mathrm{g} / \mathrm{mL})$ of SPN1, SPN2, SPN3, and SPN4 and for a period of $24 \mathrm{~h}$; following which cells were washed with PBS and incubated with drug-free medium for 3 days. The MTT reagent $(5 \mathrm{mg} / \mathrm{mL})$ was then added to each well and cells were incubated for $2 \mathrm{~h}$, followed by the addition of ethanol:DMSO (1:1) solution and optical density (OD) was measured at $540 \mathrm{~nm}$. The percentage of viable cells was calculated as follows:

$$
\begin{aligned}
\text { Cell Viability }(\%)= & \mathrm{OD}_{540 \mathrm{~nm}} \text { of treated cells } \\
& \left./ \mathrm{OD}_{540 \mathrm{~nm}} \text { of untreated cells }\right) \times 100
\end{aligned}
$$

In Vivo PA Imaging. A detailed description of the PA tomography system used for the in vivo experiments can be found elsewhere. ${ }^{17}$ Briefly, the system comprises a $50 \mathrm{~Hz}$ tunable fiber-coupled Q-switched Nd:YAG pumped OPO laser (premiScan, GWU and Quanta-Ray PRO-270, Newport Spectra Physics) as an excitation source and a Fabry-Perot based ultrasound detection system with a $-3 \mathrm{~dB}$ bandwidth of $22 \mathrm{MHz}$. In backward mode operation, photoacoustic waves are generated in tissue by the absorption of the nanosecond optical pulses provided by the OPO laser system. These waves are mapped in 2-D by raster-scanning a cw focused interrogation laser beam across the sensor and recording the acoustically induced modulation of the reflectivity at each scan point. A 12week-old SCID mouse was anaesthetized using isoflurane in oxygen $[4 \%(\mathrm{v} / \mathrm{v})$ at a flow rate of $2 \mathrm{~L} / \mathrm{min}$ for induction and $1.5 \%(\mathrm{v} / \mathrm{v})$ at a flow rate of $1 \mathrm{~L} / \mathrm{min}$ for maintenance] before being placed on a custom designed cradle on the scanner. Acoustic coupling between the mouse and the sensor was maintained by ultrasound gel applied to the surface of the mouse skin. PA signals of the vasculature were acquired at ten wavelengths between 600 and $1000 \mathrm{~nm}$, including the peak absorption wavelength of SPN4 at $800 \mathrm{~nm}$, before a subcutaneous injection of a $10 \mu \mathrm{L}$ solution containing $1.6 \mu \mathrm{g}$ of SPN4 into the right flank. PA signals were then acquired at the same wavelengths, beginning $10 \mathrm{~min}$ after injection. The placing of the mouse on a cradle ensured that same field of view $(14 \times 14 \mathrm{~mm}$ area) was imaged pre- and post-injection of SPN4. Data acquisition at each wavelength took approximately 7 min. The last of the PA signals was therefore completed within $1.5 \mathrm{~h}$ after injection of SPN4. Three-dimensional photoacoustic images were reconstructed from the detected PA signal waveforms, using a time reversal based algorithm which includes compensation for acoustic attenuation. ${ }^{18,19}$

\section{ASSOCIATED CONTENT}

\section{S Supporting Information}

The Supporting Information is available free of charge on the ACS Publications website at DOI: 10.1021/acs.bioconjchem.7b00185.

Synthesis and characterization of INDT-T 2, INDT-S 3, and INDT-BT 4 (PDF)

\section{AUTHOR INFORMATION}

\section{Corresponding Authors}

*E-mail: h.bronstein@ucl.ac.uk.

*E-mail: a.b.tabor@ucl.ac.uk.

ORCID

Alethea B. Tabor: 0000-0001-8216-0347 


\section{Present Address}

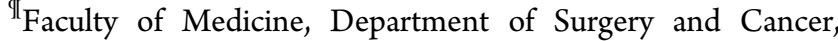
Division of Cancer, Imperial Centre for Translational and Experimental Medicine (ICTEM), Imperial College London, Hammersmith Hospital Campus, Du Cane Road, London W12 ONN, UK.

Notes

The authors declare no competing financial interest.

\section{ACKNOWLEDGMENTS}

This work was conducted within the King's College LondonUCL Comprehensive Cancer Imaging Centre (CCIC) supported by Cancer Research UK and the EPSRC, in association with MRC and DoH (UK). The authors would like to thank Cancer Research UK and the EPSRC (grants C1519/A6906 (P.J.) and C5255/A15935 (R.B.)) for financial support.

\section{DEDICATION}

This paper is dedicated to Professor Stuart L Schreiber on the occasion of his 60th birthday.

\section{ABBREVIATIONS}

DMEM, Dulbecco's Modified Eagle Medium; DPPC, 1,2dipalmitoyl-sn-glycero-3-phosphocholine; EPR, enhanced permeability and retention; GNR, gold nanorods; INDT, 2,9dihydroxy-7,14-di(thiophen-2-yl)-diindolo[3,2,1-de:3', $\left.2^{\prime}, 1^{\prime}-i j\right]$ [1,5]naphthyridine-6,13-dione; MTT, 3-(4,5-dimethylthiazol-2yl)-2,5-diphenyltetrazolium bromide; NIR, near-infrared range; PA, photoacoustic; PBS, phosphate-buffered saline; PCPDTBT, poly [cyclopentadithiophene-alt-benzothiadiazole; SPN, semiconducting polymer nanoparticles; SWCNT, single walled carbon nanotubes

\section{REFERENCES}

(1) Beard, P. (2011) Biomedical photoacoustic imaging. Interface Focus 1, 602-631.

(2) Zackrisson, S., van der Ven, S. M. W. Y., and Gambhir, S. S. (2014) Light In and Sound Out: Emerging Translational Strategies for Photoacoustic Imaging. Cancer Res. 74, 979-1004.

(3) Jathoul, A. P., Laufer, J., Ogunlade, O., Treeby, B., Cox, B., Zhang, E., Johnson, P., Pizzey, A. R., Philip, B., Marafioti, T., et al. (2015) Deep in vivo photoacoustic imaging of mammalian tissues using a tyrosinase-based genetic reporter. Nat. Photonics 9, 239-246.

(4) Zhang, J., Qiao, Z., Yang, P., Pan, J., Wang, L., and Wang, H. (2015) Recent Advances in Near-Infrared Absorption Nanomaterials as Photoacoustic Contrast Agents for Biomedical Imaging. Chin. J. Chem. 33, 35-52.

(5) Geng, J., Sun, C., Liu, J., Liao, L.-D., Yuan, Y., Thakor, N., Wang, J., and Liu, B. (2015) Biocompatible Conjugated Polymer Nanoparticles for Efficient Photothermal Tumor Therapy. Small 11, 16031610.

(6) Feng, L., Zhu, C., Yuan, H., Liu, L., Lv, F., and Wang, S. (2013) Conjugated polymer nanoparticles: preparation, properties, functionalisation and biological applications. Chem. Soc. Rev. 42, 6620-6633.

(7) $\mathrm{Xu}, \mathrm{X}$., Liu, R., and Li, L. (2015) Nanoparticles made of $\pi$ conjugated compounds targeted for chemical and biological applications. Chem. Commun. 51, 16733-16749.

(8) $\mathrm{Pu}, \mathrm{K}$., Shuhendler, A. J., Jokerst, J. V., Mei, J., Gambhir, S. S., Bao, Z., and Rao, J. (2014) Semiconducting polymer nanoparticles as photoacoustic molecular imaging probes in living mice. Nat. Nanotechnol. 9, 233-239.

(9) $\mathrm{Pu}, \mathrm{K}$. , Mei, J., Jokerst, J. V., Hong, G., Antaris, A. L., Chattopadhyay, N., Shuhendler, A. J., Kurosawa, T., Zhou, Y., Gambhir, S. S., et al. (2015) Diketopyrrolopyrrole-Based Semi- conducting Polymer Nanoparticles for In Vivo Photoacoustic Imaging. Adv. Mater. 27, 5184-5190.

(10) Liu, J., Geng, J., Liao, L.-D., Thakor, N., Gao, X., and Liu, B. (2014) Conjugated polymer nanoparticles for photoacoustic vascular imaging. Polym. Chem. 5, 2854-2862.

(11) Zha, Z., Deng, Z., Li, Y., Li, C., Wang, J., Wang, S., Qu, E., and Dai, Z. (2013) Biocompatible polypyrrole nanoparticles as a novel organic photoacoustic contrast agent for deep tissue imaging. Nanoscale 5, 4462-4467.

(12) Fallon, K. J., Wijeyasinghe, N., Yaacobi-Gross, N., Ashraf, R. S., Freeman, D. M. E., Palgrave, R. G., Al-Hashimi, M., Marks, T. J., McCulloch, I., Anthopoulos, T. D., et al. (2015) A Nature-Inspired Conjugated Polymer for High Performance Transistors and Solar Cells. Macromolecules 48, 5148-5154.

(13) Fallon, K. J., Wijeyasinghe, N., Manley, E. F., Dimitrov, S. D., Yousaf, S. A., Ashraf, R. S., Duffy, W., Guilbert, A. A. Y., Freeman, D. M. E., Al-Hashimi, M., et al. (2016) Indolo-naphthyridine-6,13-dione thiophene (INDT) building block for conjugated polymer electronics: Molecular origin of ultra-high n-type mobility. Chem. Mater. 28, 83668378.

(14) Bertrand, N., Wu, J., Xu, X., Kamaly, N., and Farokhzad, O. C. (2014) Cancer nanotechnology: The impact of passive and active targeting in the era of modern cancer biology. Adv. Drug Delivery Rev. $66,2-25$.

(15) Stahl, T., Allen, T., and Beard, P. (2014) Characterization of the thermalisation efficiency and photostability of photoacoustic contrast agents. Proceedings of SPIE, Photons Plus Ultrasound: Imaging and Sensing (Oraevsky, A. A., and Wang, L. V., Eds.) pp $89435 \mathrm{H}-1$ 89435H-8, Vol 8943, San Francisco.

(16) Weber, J., Beard, P. C., and Bohndiek, S. E. (2016) Contrast agents for molecular photoacoustic imaging. Nat. Methods 13, 639650.

(17) Zhang, E., Laufer, J., and Beard, P. (2008) Backward-mode multiwavelength photoacoustic scanner using a planar Fabry-Perot polymer film ultrasound sensor for high-resolution three-dimensional imaging of biological tissues. Appl. Opt. 47, 561-577.

(18) Treeby, B. E., and Cox, B. T. (2010) K-Wave: MATLAB Toolbox for the Simulation and Reconstruction of Photoacoustic Wave Fields. J. Biomed. Opt. 15, 021314.

(19) Treeby, B. (2013) Acoustic attenuation compensation in photoacoustic tomography using time-variant filtering. J. Biomed. Opt. 18,036008 